methods and data source development for poisoning prevention.

Methods Scoping review of peer-reviewed observational studies published between 1960 and 2019 reporting acute drug and chemical poisonings in a defined cohort within Australia using Embase, MEDLINE and Informit.

Results We identified 11,038 articles and 394 were included. Almost half the studies had a population from a single city/ district. Most studies focused on opioids (25\%), paracetamol $(9 \%)$ and amphetamines (8\%). Age and sex (>80\%) were well reported. Ethnicity, geographical remoteness and setting of exposure were rarely reported $(<5 \%)$. Individual substance was reported in two-thirds of studies but product, dose and route was rarely reported $(<10 \%)$. No improvements in reporting were seen over time and few studies used linked data. Data sources included: coronial (29\%), hospital medical records (23\%), poisons centres (20\%), toxicology units (20\%), administrative mortality data (14\%).

Conclusions We found gaps in understanding of who was affected by poisoning and environmental information on where the exposure occurred. A comprehensive understanding of the agent responsible for poisoning is poorly understood due to codeset limitations in datasets, except for a few substances. Even for those, limited information is available on the product, dose and route which has implications for control.

Learning Outcomes Policymakers, data custodians and researchers in poisoning epidemiology should prioritise improvements in known deficiencies such as creating a national minimum dataset.

\section{G.003 FEND (FULL ENERGY, NO DRUGS), AN INNOVATIVE APPROACH TO YOUTH DRUG PREVENTION}

Jacqueline Burgess*, Tom Coderre*. Preventum Initiative, Inc., Black Mountain, USA

\subsection{6/injuryprev-2021-safety.60}

Context The recreational use of prescription medications (such as opioids and benzodiazepines) and illicit drugs (methamphetamine and illegally made fentanyl) amongst adolescents is increasing, so too are accidental teen overdose statistics. To address this issue we developed FEND (Full Energy, No Drugs), a targeted primary prevention tool for youth delivered via a smartphone app.

Process Between August-November 2019, in Rhode Island, USA, we undertook a FEND pilot, targeting teens aged 14 to 19. The campaign sought to increase participants' knowledge, attitudes and beliefs (KAB) around substance misuse, addiction and overdose, using pre and post-test surveys to determine effectiveness.

Results A total of 1027 adolescents completed the baseline (pre-test) survey; 599 (58\%) completed the post-test survey. Pre and post-test analysis showed increase KAB around perceived risks of substance use $(\mathrm{P}<0.001)$; recognizing overdose symptoms $(\mathrm{P}<0.001)$; overdose response $(\mathrm{P}<0.001)$; counterfeit drugs and medication/alcohol mixing risks $(\mathrm{P}<0.001)$; awareness of drug dependence and addiction $(\mathrm{P}<0.001)$; and participants were more likely to talk with friends/family about drug risks $(\mathrm{P}<0.001)$. The percentage of correct answers between pre and post surveys increased significantly $(\mathrm{P}<0.001)$.

Learning Outcomes These results and the information gathered demonstrates the efficacy of a gamified app to engage and inform youth about commonly misused prescription medications and illicit drugs. Given the high ownership of smartphones by young people globally, and the low cost and extensive reach of smartphone apps in delivering content to, and engaging with targeted populations, results from this pilot highlight potential for technology-driven drug prevention and public health campaigns in the future.

\section{G.004 P2/N95 V'S MASKS AS PROTECTION AGAINST PUBLIC HEALTH ISSUES: TIME FOR ACTION}

'Jane Whitelaw*, ${ }^{2}$ Kate Cole, ${ }^{3}$ Peter Knott. ${ }^{1}$ University of Wollongong, Australia; ${ }^{2}$ Cole Health, Sydney, Australia; ${ }^{3}$ GCG, Australia

\subsection{6/injuryprev-2021-safety.61}

Context There has been a surge in public demand over the 2019/2020 Australian Summer to use respiratory protection against poor air quality from the extensive bushfires and more recently against the transmission of SARS $-\mathrm{CoV}-2$.

Analysis This demand has created several issues including:

1. Uncertified and non fit-for-purpose products flooding the international marketplace via direct advertising;

2. Insufficient information being available to support members of the public in how to use face masks correctly, further underpinned by frequent images of incorrect usage of face masks across popular media;

3. Use of respiratory protection by sections of the general population for which respirators were not designed for i.e. children and those with pre-existing respiratory disorders. In the general population, the use of respirators designed for healthy adults may result in adverse effects on susceptible populations.

4. Concerns that use of face masks are not appropriate for certain public health hazards, and that their use can actually increase the risk of exposure (Bin-Reza et al 2012, Huang and Morawska, 2019); and

5. A severe shortage of approved products (P2/N95) for those at high risk such as firefighters and health care workers.

Outcomes This presentation explores the different types of Respiratory Protection Devices and systematically reviews the scientific evidence of their efficacy against these Public Health respiratory hazards. Evidence-based recommendations are made and a novel infographic will be presented for use as a community engagement and education tool.

A call is also made for a publically available online register of approved products.

\section{A - Road - Data, March 23, 2021}

\section{A.001 PATTERN OF FATAL AND NON-FATAL ROAD TRAFFIC INJURIES (RTIS) IN BANGLADESH}

Abu Talab*, Salim Mahmood Chowdhury, Aminur Rahman, Shafkat Hossain, AlAmin Bhuiyan, Saidur Rahman Mashreky, Fazlur Rahman. Centre For Injury Prevention and Research, Bangladesh, Dhaka, Bangladesh

\subsection{6/injuryprev-2021-safety.62}

Backgrounds WHO estimated that yearly RTIs cause 1.35 million deaths globally. Almost 90\% of all RTI deaths occur in 
LIMCs. RTI is one of the leading causes of fatal and non-fatal in Bangladesh.

Methods The nationwide a cross sectional survey was conducted between March-June 2016 using a pretested semi-structured questionnaire. Multistage cluster sampling method considering probability-proportional-to-size strategy was used in the surveys to obtain the desired sample. Verbal autopsy method was used to ascertain the cause of death.

Result The mortality and morbidity rates due to RTI were 14.37(95\%CI;10.67-19.35) and 2164.32(95\%CI;2113.00$2217.00)$ per 100,000 per year respectively. The highest incidence rate of fatal was recorded as 21.89(95\%CI;09.3551.25 ) along with the age group $\geq 60$ years and the highest incidence of non-fatal was recorded as $2702.51 \quad(95 \%$ CI;2618-2789) along with the age group from 25 to 59 years. The fatal and non-fatal rates were significantly higher among males 22.79(16.32-31.85) compare to females 6.03 (3.17-11.47) and urban 16.00(9.99-25.63) compare to rural areas 13.47(09.19-19.74). The highest rate of fatal and nonfatal injury occurred in day time between $9.00 \mathrm{am}-12: 00 \mathrm{pm}$ and $12.0 \mathrm{pm}-15.00 \mathrm{pm}$ respectively. Usage of seatbelts was $2.1 \%$ among the drivers and only $28 \%$ motorcyclists used helmets. Talking with mobile phone was $4.50 \%$ and drug addicted was $3.8 \%$ among driver and motorcyclist at the time of accident.

Conclusion The magnitude of fatal and non-fatal RTIs was remarkably high in Bangladesh. People aged 25 to 60 years were the most vulnerable group.

Learning Outcome A country-specific strategy and interventions are needed to reduce road traffic injury burden in Bangladesh.

\section{A.002 RAPID ASSESSMENT OF VULNERABILITY OF PHILIPPINE LOCAL GOVERNMENTS TO ROAD CRASH}

${ }^{1}$ Engr. Ray Adrian Macalalag*, May Ann Sta. Lucia2. 'Department of Public Works and Highways, Iloilo City, Philippines; ${ }^{2}$ Department of Health, Iloilo City, Philippines

10.1136/injuryprev-2021-safety.63

Road safety has long been a worldwide challenge. In fact, the World Health Organization (WHO) has noted a 1.25-million death toll in 2010 or one person losing his life every 25 seconds which prompted them to raise awareness in road safety by declaring 2010-2020 as the Decade of Action for Road Safety. In the Philippines, 10,379 deaths were recorded in WHO's latest World Status Report in Global Road Safety in 2015 with the low-income group dominating its number. With regards to national policies, the Department of Transportation Road Safety Management group are implementing the speed limit law, motorcycle helmet law, drug-driving law, drink-driving law, seatbelt law, and mobile phones while driving law.

With Western Visayas in focus, the program looks into three factors namely, number of fatal and injury-resulting road crashes, national primary and secondary road density, as well as review of the said laws. It aims to determine and classify high risk cities and municipalities in Western Visayas through a tool called locational vulnerability score (LoVS). The tool will be applicable to all cities and municipalities in the Philippines provided initial data is provided to which local governments can use to assess their programs toward eradicating cases of road crashes.

\section{A.003 ROAD TRAFFIC AND DROWNING MORTALITY IN AN AFRICAN COUNTRY: A 30-YEAR PERIOD}

${ }^{1}$ Anne Abio*, 2,3 Pascal Bovet, ${ }^{3}$ Joachim Didon, ${ }^{4}$ Till Bärnighausen, ${ }^{1}$ Masood Ali Shaikh, ${ }^{5}$ Jussi P Posti, ${ }^{1,4}$ Michael Lowery Wilson. ${ }^{1}$ Injury Epidemiology and Prevention Research Group, Turku Brain Injury Centre, Division of Clinical Neurosciences, Turku University Hospital and University of Turku, Finland; ${ }^{2}$ Institute of Social and Preventive Medicine (IUMSP), Lausanne University Hospital, Lausanne, Switzerland; ${ }^{3}$ Ministry of Health, Seychelles; ${ }^{4}$ Injury Epidemiology and Prevention Unit, Heidelberg Institute of Global Health, University of Heidelberg, Heidelberg, Germany; ${ }^{5}$ Department of Neurosurgery and Turku Brain Injury Centre, Division of Clinical Neurosciences, Turku University Hospital and University of Turku, Finland

\subsection{6/injuryprev-2021-safety.64}

Background Road traffic crashes and drowning are among the leading causes of injury mortality among youth, especially in low- and middle-income countries. The aim of this population-based study was to examine trends in road traffic and drowning related mortality from 1989 to 2018 in Seychelles. Methodology The population civil death register was used to identify cases. Coding was done according to the ICD-10 codes for external causes of mortality. Mortality rates were estimated using the crude and age standardised rates. The WHO standard population was used to standardise the rates. Negative binomial regression was used to estimate the trends and annual percent changes over time.

Results Drowning and road traffic injuries accounted for approximately $21.9 \%$ and $17.5 \%$ of all injury-related deaths. Males had a higher risk both for drowning (RR 6.14, 95\% CI 3.92, 9.62; p<0.001) and road traffic injury mortality (RR 2.25, 95\% CI 1.43, 3.53; p<0.001). The drowning age standardised mortality rate was 25.9 per 100000 personyears, and road traffic mortality was 18.0 per 100000 person-years among males; and correspondingly 3.4 per 100000 and 4.6 per 100000 person-years among females, respectively. The drowning mortality increased by less than $0.01 \%$, while the road traffic mortality increased by $2.7 \%$ among males.

Conclusion The major cause of mortality was drowning. However, the annual increase in the road traffic mortality was higher during the 30-year period.

Learning outcome Policies to reduce the road traffic crashes need to be actively implemented to reduce related mortality in order to achieve the SDG target.

\section{A.004 INTERNET-BASED TEXTUAL BIG DATA AND ROAD TRAFFIC INJURIES}

${ }^{1}$ Peixia Cheng ${ }^{*}$, ${ }^{2}$ Jianxin Wang, ${ }^{1}$ Wangxin Xiao, ${ }^{3}$ David Schwebel, ${ }^{1}$ Peishan Ning, ${ }^{4}$ Yue Wu, ${ }^{1}$ Guoging Hu. ${ }^{1}$ Department of Epidemiology and Health Statistics, Xiangya School of Public Health, Central South University, Changsha, China; ${ }^{2}$ School of Computer Science and Engineering, Central South University, Changsha, China; ${ }^{3}$ Department of Psychology, University of Alabama at Birmingham, Birmingham, USA; ${ }^{4}$ Department of Environmental and Occupational Health, Xiangya School of Public Health, Central South University, Changsha, China

\subsection{6/injuryprev-2021-safety.65}

Background Internet-based big data may offer important and timely information concerning road traffic injury data, supplementing official government statistics. We developed computerbased approaches to define, extract and automatically collect internet-based Chinese language big data on road traffic injuries. 